\title{
The Glucagon-Like Peptide 1 (GLP-1) Analogue, Exendin-4, Decreases the Rewarding Value of Food: A New Role for Mesolimbic GLP-1 Receptors
}

\author{
Suzanne L. Dickson, ${ }^{1}$ Rozita H. Shirazi, ${ }^{1}$ Caroline Hansson, ${ }^{1}$ Filip Bergquist, ${ }^{2}$ Hans Nissbrandt, ${ }^{2}$ \\ and Karolina P. Skibicka ${ }^{1}$ \\ Departments of ${ }^{1}$ Physiology and ${ }^{2}$ Pharmacology, Institute of Neuroscience and Physiology, The Sahlgrenska Academy at the University of Gothenburg, \\ SE-405 30 Gothenburg, Sweden
}

\begin{abstract}
The glucagon-like peptide 1 (GLP-1) system is a recently established target for type 2 diabetes treatment. In addition to regulating glucose homeostasis, GLP-1 also reduces food intake. Previous studies demonstrate that the anorexigenic effects of GLP-1 can be mediated through hypothalamic and brainstem circuits which regulate homeostatic feeding. Here, we demonstrate an entirely novel neurobiological mechanism for GLP-1-induced anorexia in rats, involving direct effects of a GLP-1 agonist, Exendin-4 (EX4) on food reward that are exerted at the level of the mesolimbic reward system. We assessed the impact of peripheral, central, and intramesolimbic EX4 on two models of food reward: conditioned place preference (CPP) and progressive ratio operant-conditioning. Food-reward behavior was reduced in the CPP test by EX4, as rats no longer preferred an environment previously paired to chocolate pellets. EX4 also decreased motivated behavior for sucrose in a progressive ratio operant-conditioning paradigm when administered peripherally. We show that this effect is mediated centrally, via GLP-1 receptors (GLP-1Rs). GLP-1Rs are expressed in several key nodes of the mesolimbic reward system; however, their function remains unexplored. Thus we sought to determine the neurobiological substrates underlying the food-reward effect. We found that the EX4-mediated inhibition of food reward could be driven from two key mesolimbic structures - ventral tegmental area and nucleus accumbens - without inducing concurrent malaise or locomotor impairment. The current findings, that activation of central GLP-1Rs strikingly suppresses food reward/motivation by interacting with the mesolimbic system, indicate an entirely novel mechanism by which the GLP-1R stimulation affects feeding-oriented behavior.
\end{abstract}

\section{Introduction}

The worldwide obesity epidemic has prompted an urgent need to increase understanding of the neurobiological mechanisms underpinning overeating with an ambition to develop effective treatments for obesity and eating disorders. Eating beyond immediate metabolic need is a key component of weight gain leading to obesity. High-calorie palatable foods can reinforce their own intake by increasing their rewarding value and by increasing the pleasure associated with eating (Zheng et al., 2009a). Therefore, effective future obesity treatments should ideally impact on

\footnotetext{
Received Dec. 20, 2011; revised Feb. 14, 2012; accepted Feb. 15, 2012.

Author contributions: K.P.S. designed research; R.H.S., C.H., F.B., and K.P.S. performed research; H.N. and K.P.S. analyzed data; S.L.D. and K.P.S. wrote the paper.

We thank the Swedish Research Council for Medicine (2011-3054 to K.P.S., 2009-S266 to S.L.D., 12208, 20092618), European Commission 7th Framework [FP7-HEALTH-2009-241592 (EurOCHIP), FP7-KBBE-2009-3-245009 (NeuroFAST), and FP7-KBBE-2010-4-266408 (Full4Health)], Forskning och Utvecklingsarbete/Avtal om Läkarutbildning och Forskning Göteborg (ALFGBG-138741), NovoNordisk Fonden and the Swedish Foundation for Strategic Research to Sahlgrenska Center for Cardiovascular and Metabolic Research (A305-188). We also thank Frida Larsson for her technical assistance.

The authors declare no competing financial interests.

This article is freely available online through the J Neurosci Open Choice option.

Correspondence should be addressed to Dr. Karolina P. Skibicka, Department of Physiology/Endocrinology, Institute of Neuroscience and Physiology, The Sahlgrenska Academy at the University of Gothenburg, Medicinaregatan 11, P.0. Box 434, SE-405 30 Gothenburg, Sweden. E-mail: Karolina.Skibicka@neuro.gu.se.

DOI:10.1523/JNEUROSCI.6326-11.2012

Copyright $\odot 2012$ the authors $\quad 0270-6474 / 12 / 324812-09 \$ 15.00 / 0$
}

food-reward behaviors. The neurobiology underlying foodreward behavior is just emerging, with a clear role for established reward-associated neurotransmitters, such as dopamine, alongside recently discovered roles for nontraditional, metabolic targets that include gut hormones like ghrelin, cholecystokinin, polypeptide Y, and fat-derived leptin (see Fulton, 2010, for review).

Glucagon-like peptide 1 (GLP-1) is secreted from the intestinal tract in response to nutrients and is also produced in the nucleus of the solitary tract (NTS) of the brainstem with projections throughout the CNS. It has captivated interest due to its incretin (aiding the action of insulin) and anorectic effects (Turton et al., 1996; Holst, 2007). The GLP-1 analog, Exendin-4 (EX4), a highly selective GLP-1 receptor (GLP-1R) agonist (Thorens et al., 1993) is a rapidly emerging novel diabetes treatment. It is now also evaluated as a potential obesity treatment due to its beneficial effects to reduce food intake and body weight. Interest in GLP-1 as a potential overeating/obesity treatment is further prompted by data showing elevated levels of GLP-1 following gastric bypass, both in human patients (Laferrère et al., 2007) and in rat models (Zheng et al., 2009b). Little is known about the central mechanisms behind the anorectic effects of GLP-1. To date, research has focused primarily on the impact of this peptide on the homeostatic brain circuits that include well established areas involved in metabolic control, the arcuate and paraven- 
tricular nuclei of the hypothalamus (McMahon and Wellman, 1998), and the brainstem NTS (Hayes et al., 2009).

The GLP-1R, a stimulatory G-protein receptor (Takhar et al., 1996), is expressed in several discrete CNS nuclei including the hypothalamus and hindbrain (Merchenthaler et al., 1999). Importantly, GLP-1Rs are expressed in key brain areas controlling reward and motivated behaviors that include the ventral tegmental area (VTA) and the nucleus accumbens (NAc) (Merchenthaler et al., 1999); their role within these brain areas, however, remains largely unknown. Given the rapid and widespread use of EX4 and its potential to cross the blood-brain barrier and gain access to brain parenchyma (Kastin and Akerstrom, 2003), it is of considerable interest to determine the function of this peptide in brain areas other than those involved in homeostatic and metabolic control.

In this study we provide the first evidence that EX4 regulates food-motivated behavior and food reward. To assess the importance of central circuitry for this effect, we determined the effects of central delivery of EX4 on food motivation. We also studied the neurobiological substrates underpinning these effects by evaluating the direct action of EX4 on key mesolimbic nodes, the VTA and the NAc.

\section{Materials and Methods}

Animals. Male Sprague Dawley rats, weighing $250 \mathrm{~g}$ at the start of the experiments, were supplied by Charles River. Lights were turned on at 6:00 A.M. and off at 6:00 P.M. Experimental testing commenced at 10:00 A.M. All experiments were approved by the Göteborg University Institutional Animal Care Committee (ethical permissions 334-04 and 336-09).

Drugs. EX4 and Exendin-3 ${ }_{(9-39)}$ (EX3) were purchased from Tocris Bioscience, dissolved in saline [vehicle for intraperitoneal (IP) administration] or artificial CSF (vehicle for all central injections), and stored as aliquots in $-20^{\circ} \mathrm{C}$. EX4 is a long-lasting analog of GLP-1 and a full agonist at the GLP-1R (Thorens et al., 1993). EX3 is a selective antagonist at the GLP-1R (Thorens et al., 1993). Studies with radiolabeled EX4 showed an identical distribution pattern of binding sites as those shown with GLP-1 in the CNS (Göke et al., 1995).

Behavioral testing. To test the role of GLP-1 stimulation in foodmotivated behavior and food reward, we used two behavioral models typically used to measure behavioral properties of addictive drugs, the progressive ratio (PR) operant conditioning test (Hodos, 1961) and the conditioned place preference (CPP) paradigm. The first task is a well established test for alterations in motivated behavior (Hodos, 1961); the higher the motivation to obtain the rewarding substance, the harder the rat is willing to work (press a lever) for it. In the CPP task, the animals spend more time in an environment previously paired with a reward reinforcer, in this case, a chocolate pellet.

Operant conditioning. Food-induced operant conditioning training and testing were conducted in rat conditioning chambers $(30.5 \times 24.1 \times$ $21.0 \mathrm{~cm}$; Med-Associates). Each chamber had a metal grid floor, two retractable levers with white light bulbs above them, and a food pellet dispenser that can deliver $45 \mathrm{mg}$ of sucrose pellets (Test Diet, GlaxoSmithKline) to the food tray. Data collection and processing were controlled by MED-PC software. Rats were trained to press a lever for a 45 $\mathrm{mg}$ sucrose reward. Training: The procedure used for operant conditioning was adapted from la Fleur et al. (2007) and Skibicka et al. (2011, 2012). All rats were subjected to a mild food restriction paradigm during which their initial body weight was gradually reduced to $90 \%$ over a period of 1 week. For experiments in which cannulated rats were used, the training commenced 1 week after the surgery. Training was conducted in four stages: rats were first trained on fixed ration (FR) 1 schedule (single press on the active lever $=$ delivery of one sucrose pellet), followed by FR3 and FR5 ( 3 and 5 presses per one pellet, respectively), where a minimum of 50 responses per session on the active lever was required for the advancement to the next schedule, culminating with PR conditioning until stable responding was achieved. Operant response testing was performed after the responses stabilized (number of pellets earned per session did not differ more than $15 \%$ for three consecutive sessions). Rats received the drug injection early in the light cycle after partial ( $10 \mathrm{~g}$ of chow available) overnight food restriction (unless otherwise stated). This paradigm was chosen to avoid the confounding effect of a variable overnight food intake on the lever pressing and provide a reliable motivation to work for sucrose, which was hypothesized to be subsequently attenuated by EX4.

For each experiment, the following conditions were used: (1) For IP drug application: vehicle, $0.3 \mu \mathrm{g} / \mathrm{kg}$ EX4, $2.4 \mu \mathrm{g} / \mathrm{kg}$ EX4, injections at 1 $\mathrm{ml} / \mathrm{kg}$. (2) For third intracerebroventricular (ICV) drug application: vehicle, $0.03 \mu \mathrm{g}$ EX4, $0.1 \mu \mathrm{g}$ EX4, $0.3 \mu \mathrm{g}$ EX4, $1.5 \mu \mathrm{g}$ EX4, $3.0 \mu \mathrm{g}$ EX4, all injections in a volume of $1 \mu \mathrm{l}$. (3) For lateral ventricle EX4/EX3 combination experiment: 1 . vehicle-vehicle; 2 . vehicle- $0.2 \mu \mathrm{g}$ EX4; 3 . EX4EX3 $20 \mu \mathrm{g}$; 4. EX3-vehicle. (4) For unilateral VTA or NAc drug application: vehicle, $0.03 \mu \mathrm{g}$ EX4, $0.1 \mu \mathrm{g}$ EX4, in a volume of $0.5 \mu \mathrm{l}$. (5) For unilateral VTA subthreshold dose microinjections: vehicle, $0.01 \mu \mathrm{g}$ EX4 in a volume of $0.5 \mu \mathrm{l}$. (6) For unilateral VTA drug application in satiated rats: vehicle, $0.03 \mu \mathrm{g} \mathrm{EX4,} 0.1 \mu \mathrm{g} \mathrm{EX4}$, in a volume of $0.5 \mu \mathrm{l}$. (7) For unilateral VTA control site microinjections: vehicle, $0.1 \mu \mathrm{g}$ EX4 in a volume of $0.5 \mu$ l. (8) For unilateral VTA or NAc drug application to measure potential malaise responses: vehicle, $0.03 \mu \mathrm{g} \mathrm{EX} 4,0.1 \mu \mathrm{g} \mathrm{EX} 4$, in a volume of $0.5 \mu \mathrm{l}$.

Conditioned place preference. The CPP test was performed in satiated rats $(n=18)$ using an apparatus comprised of two connected chambers with distinct visual and tactile qualities. Initial preference for one chamber was assessed on the first day during a 20 min pretest, and the least preferred compartment was subsequently paired with rewarding/palatable food (chocolate pellets; Ms, Marabou, Kraft Foods). The preferred chamber was paired with less-rewarding food (normal chow). The pretest was followed by 20 conditioning sessions (two sessions per day). One day following the last conditioning session, rats were injected IP with vehicle (saline) or EX4 $(0.3 \mu \mathrm{g} / \mathrm{ml}$ injected at $1 \mathrm{ml} / \mathrm{kg})$ at $10 \mathrm{~min}$ before being placed in the CPP apparatus for $10 \mathrm{~min}$. The CPP test informs on how rewarding the rat finds the palatable food. During the CPP test, rats do not have access to food, enabling dissociation of the intake of palatable food from the reward evaluation process. The behavior of the animals was recorded and time spent in each compartment was determined by visual analysis of a video recording.

Chow intake and body weight. Home cage chow intake was measured after EX4 injection into the VTA or the NAc. Rats were placed in their home cages immediately after the operant conditioning experiment with preweighed chow, and chow was weighed after $1 \mathrm{~h}$ and $24 \mathrm{~h}$. Body weight measurement was taken immediately before injection and $24 \mathrm{~h}$ postinjection.

Motor activity measurements. For motor activity measurements (after VTA and NAc EX4 microinjections), rats were placed in chambers 60 min before VTA or NAc EX4 $(0.1 \mu \mathrm{g} / 0.5 \mu \mathrm{l})$ injection and $60 \mathrm{~min}$ spontaneous $\mathrm{X}-\mathrm{Y}$ plane and rearing postinjection activity were measured and analyzed in 10 min bins. Food was not available during this test.

Pica response. To investigate whether EX4 microinjection into VTA or NAc induces malaise, the pica response was measured after EX4 VTA and NAc microinjections. The pica response assesses the consumption of non-nutritive substances (here, kaolin pellets; Research Diets) following administration of potential nausea-inducing agents (Mitchell et al., 1976; Takeda et al., 1993). An increase in kaolin consumption indicates malaise. Chow intake was also measured here to confirm that the EX4 injections evaluated for pica were effective at reducing intake. All rats were exposed to kaolin before the test day, and all were confirmed to have tasted the compound before testing.

Brain cannulation. For behavioral studies targeting the CNS, in separate experiments, a lateral, third ventricle (ICV), VTA or NAc guide cannula was positioned and attached to the skull with dental acrylic and jeweler's screws and closed with an obturator, as described previously (Skibicka et al., 2011, 2012). Briefly: a third ventricular guide cannula (26 gauge, Plastics One; coordinates: on the midline, $2.0 \mathrm{~mm}$ posterior to bregma, and $5.5 \mathrm{~mm}$ ventral to dura mater, with injector aimed $7.5 \mathrm{~mm}$ ventral to the dura); lateral ventricular guide cannula (coordinates: \pm 1.6 $\mathrm{mm}$ from the midline, $0.9 \mathrm{~mm}$ posterior to bregma, and $2.0 \mathrm{~mm}$ ventral 
to dura mater, with injector aimed $4.0 \mathrm{~mm}$ ventral to the dura); VTA guide cannula $[ \pm 0.75 \mathrm{~mm}$ from the midline, $5.7 \mathrm{~mm}$ posterior to bregma, and $6.5 \mathrm{~mm}$ ventral from the surface of the skull, with injector aimed $8.0 \mathrm{~mm}$ ventral to skull (for the dose control site the injector was aimed $6.5 \mathrm{~mm}$ ventral to skull)]; NAc shell guide cannula $( \pm 0.75 \mathrm{~mm}$ from the midline, $1.7 \mathrm{~mm}$ anterior to bregma, and $6.0 \mathrm{~mm}$ ventral to skull, with injector aimed $7.5 \mathrm{~mm}$ ventral) were implanted under isoflurane anesthesia. The microinjection site for VTA and NAc guide cannula was verified postmortem by microinjection of India ink at the same microinjection volume $(0.5 \mu \mathrm{l})$ used throughout the study. Only rats with the correct placement were included in the data analysis. The VTA was chosen as a target since all motivated behaviors including motivation for food are under tight control of the mesolimbic system. The VTA is the key source of dopamine neurons projecting to a range of mesocorticolimbic targets. One mechanism by which EX4 may control motivated behavior for food is by affecting the activity of VTA neurons via a direct action on the VTA. Indeed, GLP-1Rs are present in the VTA; their physiological function, however, has remained unexplored. The NAc was chosen here as it contains GLP-1Rs and it is one of the crucial terminal sites for the VTA dopamine projections that regulate reward-motivated behavior. Because dopamine transmission can also be regulated presynaptically at the sites of release and GLP-1Rs are also detected in the shell of NAc, we explored the possibility that NAc represents another mesolimbic direct target for EX4 action on motivated behavior.

Statistics. All statistical analysis was performed using GraphPad Prism software.

\section{Results}

EX4 decreases food-reward and food-motivated behavior

Rats responding for a sucrose reward ( $45 \mathrm{mg}$ pellet) under a PR reinforcement schedule (i.e., a schedule in which the number of presses required to obtain a single sucrose pellet increases progressively) were treated with a selective, long-lasting GLP-1R agonist, EX4 (0.3 and $2.4 \mu \mathrm{g} / \mathrm{kg})$ at $10 \mathrm{~min}$ before placement in the operant boxes. The doses were chosen based on Hayes et al. (2008). Intraperitoneally administered EX4 significantly decreased food-reward behavior as measured by sucrose motivation through the entire period of the test (one-way ANOVAs for each time point; 10 min: $F_{(2,109)}=30.31, p<0.0001 ; 30 \mathrm{~min}$ : $F_{(2,109)}=41.54, p<0.0001 ; 120 \mathrm{~min}: F_{(2,109)}=16.38, p<0.0001$; Figure $1 A)$.

Next we investigated the impact of GLP-1R stimulation in a complementary test of food-reward behavior - the food-induced $\mathrm{CPP}$, in which a rat shows a preference for a chamber previously paired with palatable food ( 1 g chocolate pellets) over a chamber previously paired with regular chow. Rats injected with EX4 (0.3 $\mu \mathrm{g} / \mathrm{kg}$, i.p.) at $10 \mathrm{~min}$ before the CPP test lost preference for the chocolate-paired chamber seen in the vehicle-treated group (Student's $t$ test, $p<0.05$, Fig. $1 B$ ). Overall locomotor activity during the CPP was not altered by the treatment (Fig. 1C).

Following the peripheral administration experiments, we sought to determine whether the site of action for EX4 suppression of food-reward behavior is central; rats were acutely injected centrally (ICV) with EX4 at 10 min before the sucrose reinforced PR operant conditioning. Central EX4 treatment significantly reduced operant behavior at several doses and at all time points tested (one-way ANOVA for each time point: $10 \mathrm{~min}: F_{(5,24)}=$ $7.0, p<0.0005 ; 30 \mathrm{~min}: F_{(5,24)}=7.3, p<0.0005 ; 60 \mathrm{~min}: F_{(5,24)}=$ $8.5, p<0.0005 ; 120$ min: $F_{(5,24)}=8.52, p<0.0001$; Figure 2$)$. It is important to note that GLP-1R stimulation, via both IP and ICV drug administration, has previously been shown to reduce the rate of gastric emptying (Schirra and Göke, 2005). Although during the experiments performed here the rats were food restricted, thereby reducing the chance of gastric emptying reduction contributing to observed responses, we cannot entirely
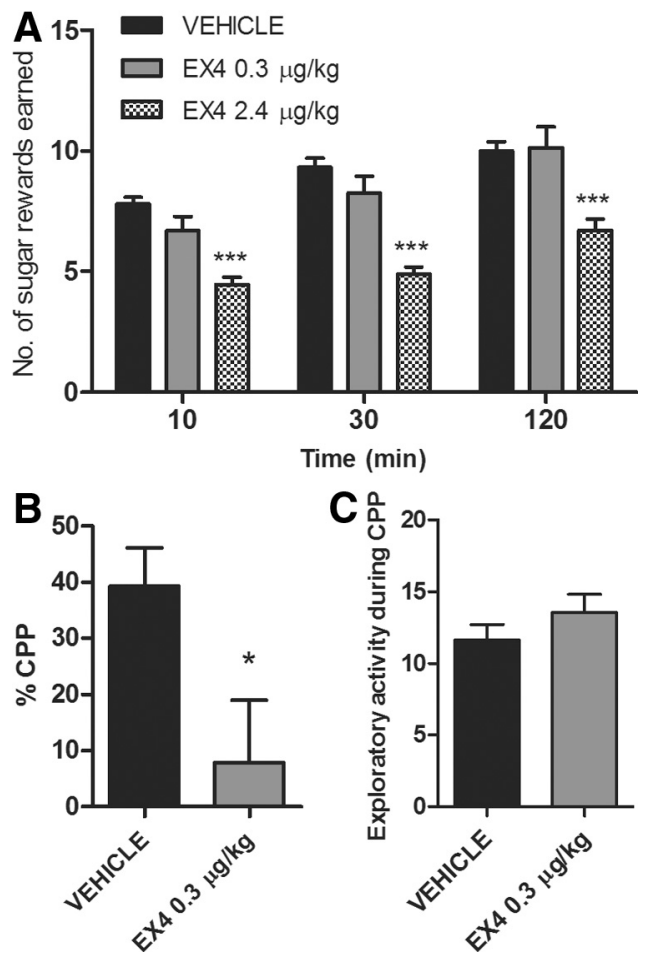

Figure 1. GLP-1 receptor stimulation with EX4 decreased food-reward behavior. The effects of intraperitoneal injection of EX4 on PR operant responding for sucrose and the ability of chocolate to condition a place preference were tested. $\boldsymbol{A}$, EX4 decreased number of sucrose rewards earned in an operant lever-pressing paradigm. ${ }^{* *} p<0.0005$, compared with vehicle using Tukey test; $n=16-48$ per group. $\boldsymbol{B}$, Preference for the chamber paired to palatable food was attenuated by EX4 treatment. The increased preference (\% (PP) was calculated using the following formula: [(test - pre-test)/(total time - pre-test)] $\times 100 .{ }^{*} p<0.05$, comparing vehicle to EX4 using Student's $t$ test. $C$, Exploratory activity was not altered by the EX4 treatment during the CPP test; $n=9$ per treatment group. Data represent mean \pm SEM.

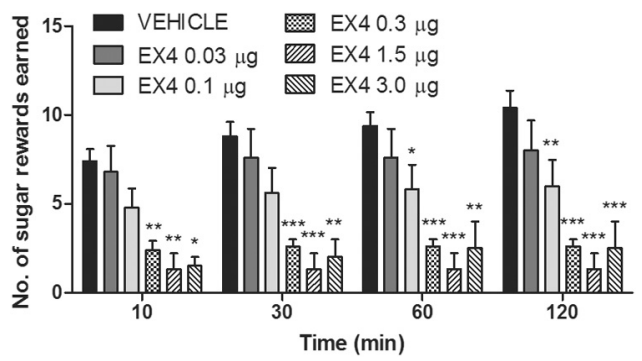

Figure 2. Central (ICV) administration of EX4 is sufficient to decrease food-motivated behavior in PR operant responding for a sucrose reward paradigm. ${ }^{*} p<0.05$, ${ }^{* *} p<0.005$, ${ }^{* * *} p<0.0005$ comparing vehicle to each EX4 dose using the Tukey test after obtaining a significant main effect by one-way ANOVA for each time point; $n=2-10$. Data represent mean \pm SEM

eliminate the possibility that reduced emptying rate induced by EX4 has contributed to effects observed.

Collectively, the data from the CPP and operant-responding tests support a striking role for EX4 stimulation in food-reward behavior.

\section{Central EX4 decreases food-motivated behavior through GLP-1R stimulation}

To confirm that the reduction in food-motivated behavior by EX4 is mediated through the CNS GLP-1R, the impact of pretreatment with a selective GLP-1R antagonist (EX3, $20 \mu \mathrm{g}$ ICV in $1 \mu \mathrm{l}$; Thorens et al., 1993) on central EX4-induced $(0.2 \mu \mathrm{g}$ in $1 \mu \mathrm{l}$, 


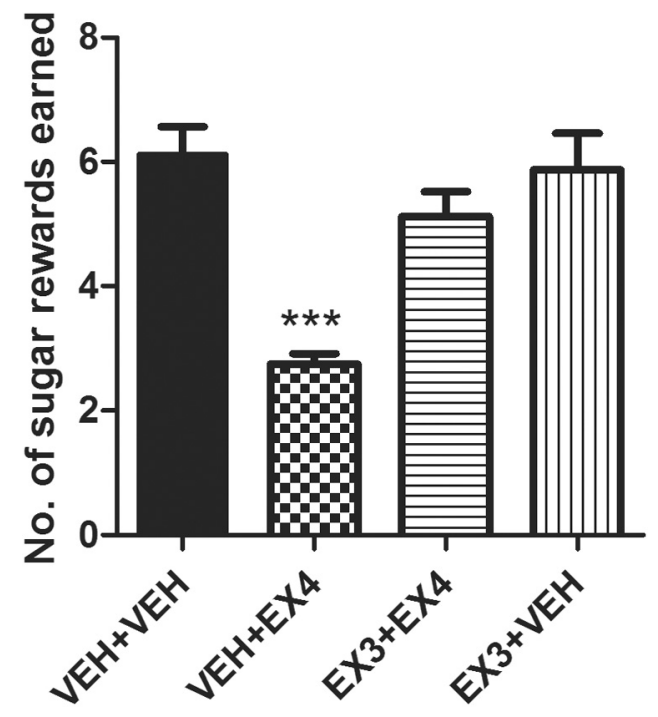

Figure 3. Central EX4 reduction in food-reward behavior is mediated via GLP-1Rs as pretreatment with a selective GLP-1R antagonist, EX3, abolishes the suppressive effect of EX4 on PR operant conditioning. ${ }^{* *} p<0.0005$ comparing vehicle to EX4 using the Tukey test after detecting a significant interaction of pretreatment with EX4 in two-way ANOVA and a main effect of one-way ANOVA; $n=8$. Data represent mean \pm SEM.

ICV) reduction in sucrose operant behavior was tested. Two-way ANOVA revealed a significant interaction between pretreatment $\times \operatorname{EX} 4\left(F_{(1,28)}=9.62, p<0.005\right.$; Fig. 3$)$. The dose of EX3 chosen did not produce effects per se when administered alone, enabling a reliable interpretation of an interaction with EX4. An effect of EX3 alone was also unlikely in this paradigm given that rats were partially food restricted, a state of low endogenous GLP-1 levels. The dramatic reduction in the number of rewards earned with EX4 (post hoc Tukey test, $p<0.0001$ ) was abolished by central EX3 pretreatment.

\section{The VTA is a direct site of action for EX4-driven food-motivated behavior and food intake}

To test whether the VTA is a target for the regulation of food reward by EX4, we assessed the effects of microinfusion of EX4 $(0.01,0.03$, and $0.1 \mu \mathrm{g}$ in $0.5 \mu \mathrm{l})$ into the VTA on sucrosemotivated behavior in the PR operant responding paradigm. Based on the ICV study results, the doses were chosen to be subthreshold (0.01 and $0.03 \mu \mathrm{g})$ and just suprathreshold $(0.1 \mu \mathrm{g})$ when administered ICV. VTA EX4 treatment significantly reduced operant behavior at 0.03 and $0.1 \mu \mathrm{g}$ doses and at all time points tested (one-way ANOVA, 10 min: $F_{(2,14)}=8.8, p<0.005$; $30 \mathrm{~min}: F_{(2,14)}=9.3, p<0.001 ; 60 \mathrm{~min}: F_{(2,14)}=10.7, p<0.0005$; 120 min: $F_{(2,14)}=8.8, p<0.005$; Figure $4 C$ ) without producing significant changes in activity at the inactive lever (data not shown). The lowest dose $(0.01 \mu \mathrm{g})$ was tested in a separate group of rats. Even though strong trends were detected $(n=7 ; 7.4 \pm 0.4$ vs $5.2 \pm 1.0$ for vehicle and EX4, respectively, $p=0.07$ on $1 \mathrm{~h}$ chow intake and $9.3 \pm 0.7$ vs $8.8 \pm 0.6, p=0.14$ on $120 \mathrm{~min}$ rewards earned), no significant effect of this dose was noted. Thus $0.03 \mu \mathrm{g}$ was the lowest effective dose used for VTA injection. To confirm that the effects of EX4 were specific to the VTA and not due to nonspecific diffusion of the drug to surrounding regions, we microinjected the highest dose of EX4 tested in the VTA $(0.1$ $\mu \mathrm{g}$ in $0.5 \mu \mathrm{l}$ ) in a site $2.0 \mathrm{~mm}$ dorsal to the VTA. This control site was chosen based on the assessment of the ink diffusion in the VTA targeted injections. The small amount of ink rarely found outside of the VTA was located dorsally to the VTA. No effects of
EX4 were detected in that paradigm $(n=6 ; 8.5 \pm 0.5$ vs $7.4 \pm 0.4$ for vehicle and EX4, respectively, $p=0.3$ on $1 \mathrm{~h}$ chow intake and $9.7 \pm 1.2$ vs $9.5 \pm 1.3, p=0.14$ on 120 min rewards earned). This highlights the specificity of the injection by demonstrating that the highest dose effective in both the VTA and the NAc in reducing reward behavior and chow intake was not effective when microinjected into a control region $2.0 \mathrm{~mm}$ dorsal to the VTA.

Given the uncharted nature of the VTA GLP-1R population and the established role of GLP-1 in the control of food intake, we also assessed chow intake behavior immediately subsequent to operant testing for a period of $1 \mathrm{~h}$ and $24 \mathrm{~h}$. Both the $1 \mathrm{~h}$ (one-way ANOVA, $F_{(2,15)}=7.8, p<0.005$; Fig. $4 B$ ) and the $24 \mathrm{~h}$ (one-way ANOVA, $F_{(2,15)}=111.6, p<0.0001$; see Fig. $6 A$ ) chow intake were significantly decreased in animals microinjected with EX4 into the VTA. Only the higher dose produced a significant response at $1 \mathrm{~h}$ (post hoc Tukey test, $p<0.005$ ), although both doses were effective at the $24 \mathrm{~h}$ time point. Body weight was significantly reduced by the EX4 VTA treatment at the $24 \mathrm{~h}$ time point (one-way ANOVA, $F_{(2,15)}=83.3, p<0.0001$; Fig. $6 B$ ).

We also determined, in a separate experiment, the effect of VTA EX4 injections on $60 \mathrm{~min}$ locomotor activity and exploration (rearing) activity outside of the operant testing chambers, without access to food. Neither locomotor activity nor rearing was altered by VTA EX4 microinjection (Fig. $4 D, E$ ) at a dose that significantly decreased both chow intake and operant behavior when microinjected into the VTA. Collectively, these data indicate that VTA GLP-1R signaling plays a pivotal role in food reward and food intake.

Food-motivated behavior, food intake, and spontaneous activity are altered by microinjection of EX4 into the NAc To determine whether NAc GLP-1-expressing cells are a direct target for EX4, we microinjected EX4 $(0.03$ and $0.1 \mu \mathrm{g}$ in $0.5 \mu \mathrm{l})$ into the shell of the NAc and, as above, measured PR operant behavior for sucrose, chow intake and, in a separate test, spontaneous locomotor activity. Direct NAc GLP-1R stimulation with EX4 suppressed operant responding at all time points (one-way ANOVAs, 10 min: $F_{(2,15)}=3.8, p<0.05 ; 30 \mathrm{~min}: F_{(2,15)}=7.2$, $p<0.005 ; 60 \mathrm{~min}: F_{(2,15)}=8.2, p<0.005 ; 120 \mathrm{~min}: F_{(2,15)}=10.0$, $p<0.0005$; Fig. $5 C$ ) without producing significant changes in activity at the inactive lever (data not shown). In contrast to the results obtained for VTA microinjection, only the higher dose produced a significant change in motivated behavior for sucrose, suggesting that this nucleus is less sensitive to EX4 effects on food reward. Chow intake was also decreased in the EX4 treatment group, only with the higher dose at $1 \mathrm{~h}\left(F_{(2,14)}=6.9, p<0.005\right.$; Fig. $5 B)$ and $24 \mathrm{~h}$ time point $\left(F_{(2,15)}=9.8, p<0.0005\right.$; Fig. $\left.6 C\right)$. No significant body weight reduction was found at $24 \mathrm{~h}$ postinjection (Fig. $6 D$ ). In contrast to effects of EX4 microinjection into the VTA, NAc EX4 reduced locomotor activity and rearing for a $10 \mathrm{~min}$ period, $10 \mathrm{~min}$ postinjection $(p<0.05$, Fig. $5 D, E)$. Locomotor activity during the remaining $50 \mathrm{~min}$ was not altered by the treatment. Changes in locomotor activity are often used in addiction research as a correlate of dopamine release from VTA into the NAc (Pijnenburg and van Rossum, 1973). Orexigenic ghrelin, for example, when injected into the VTA, clearly elevates locomotor activity and accumbal dopamine release (Jerlhag et al., 2007). Therefore, the brief reduction in locomotor activity found here after NAc GLP-1R stimulation might be indicative of a depression of the NAc dopamine signal. Although left-lever activity after NAc EX4 stimulation was not affected, and the reduction in locomotor activity detected in a separate locomotor test was short lasting (10 min period only), we cannot entirely eliminate the 
A
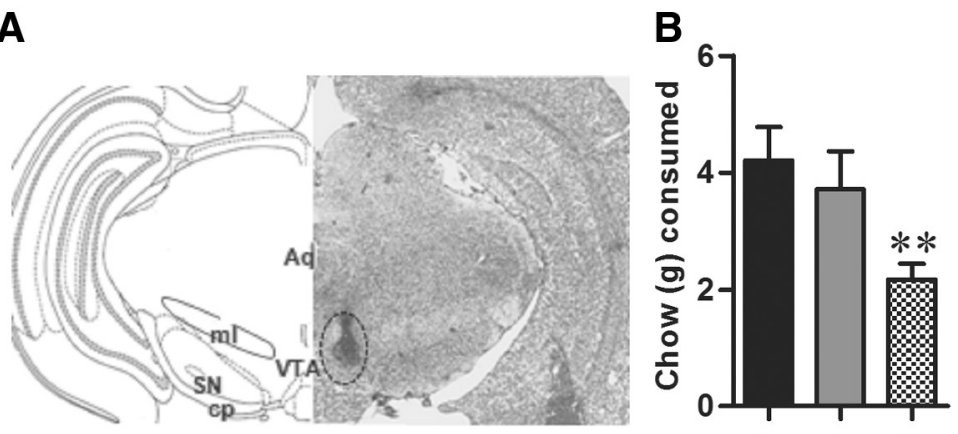

C

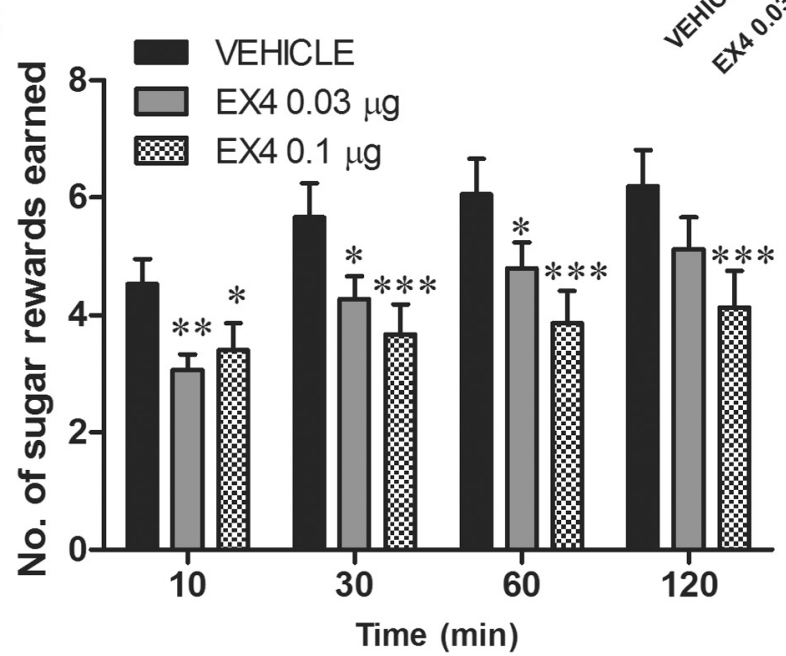

D

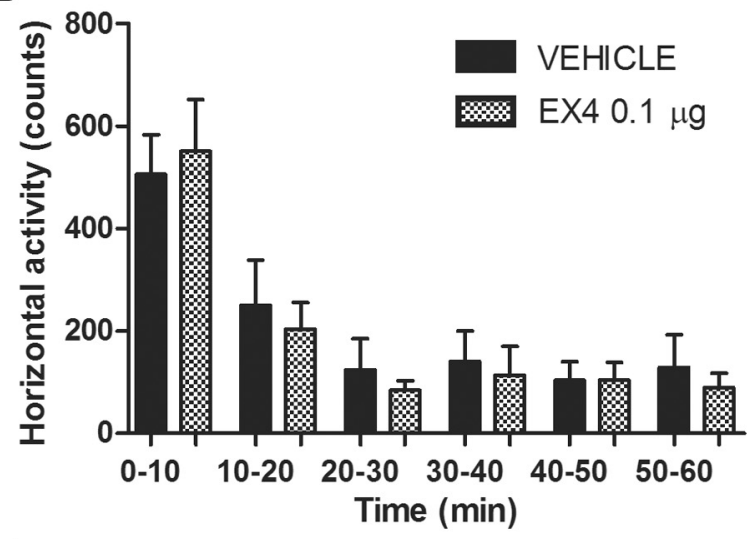

E

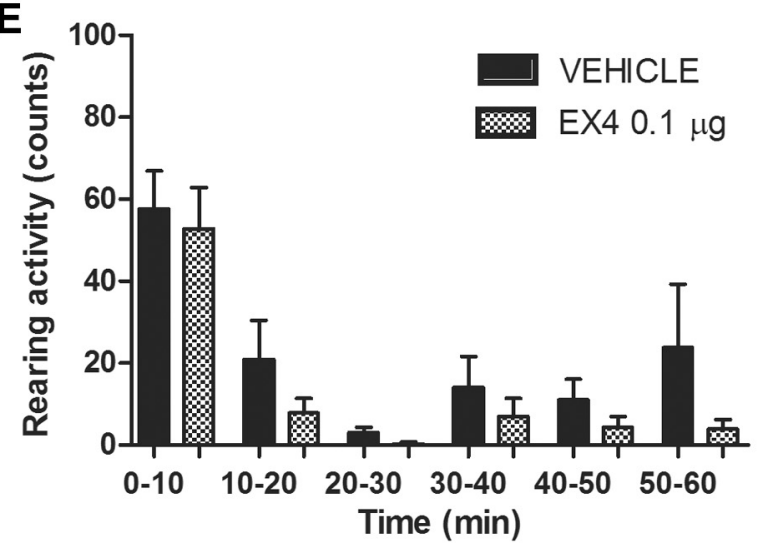

possibility that this small effect on motor activity contributed to the reduction in rewards earned. The differential effect on activity in the VTA versus NAc also further supports the site specificity of the EX4 injection.

The VTA is a direct site of action for EX4-driven food-motivated behavior and food intake in satiated state

To determine whether the food restriction used in the previous paradigms to enhance the food motivation response is required for the reward suppressing and anorexic effects of intra-VTA EX4, food motivation and food intake effects of EX4 microinjected into the VTA were tested in rats allowed to eat ad libitum overnight before injection. In this experimental setup, VTA EX4 treatment significantly reduced operant behavior (one-way ANOVA, 120 min: $F_{(2,13)}=5.2, p<0.05$; Fig. $7 A), 1$ h chow intake $\left(F_{(2,13)}=3.6\right.$, $p<0.05$; Fig. $7 C)$ and 24 h chow intake $\left(F_{(2,8)}=8.1, p<0.005\right.$; Fig. $\left.7 D\right)$ with the highest dose significantly reducing food reward and $1 \mathrm{~h}$ food intake, and both doses producing a reduction in $24 \mathrm{~h}$ intake. Interestingly, if the data on rewards earned by the satiated animals are analyzed with selecting those subjects that presented with a high motivation to work for sugar at their satiated baseline (on the vehicle condition earned 6 or more rewards; $n=6$ ) both doses of EX4 significantly reduce the rewards earned (oneway ANOVA, 120 min: $F_{(2,5)}=5.9, p<$ 0.05; Fig. $7 B$ ). Conversely, the subjects displaying a low motivation for sucrose (on vehicle condition earned 5 or less rewards; $n=8$ ) in a satiated state were less responsive to reward reducing effects of EX4 (one-way ANOVA, 120 min: $F_{(2,7)}=$ $2.7, p=0.10$; Fig. $7 B$ ). This could potentially indicate an enhanced sensitivity to the reward-reducing effects of EX4 in high reward responders, i.e., those rats are highly motivated to earn sugar despite their satiated state.

cerebralpeduncle; $\mathrm{SN}$, substantia nigra; $\mathrm{ml}$, medial lemniscus. $B$, VTA microinjection of EX4 produced chow anorexia. ${ }^{* *} p<$ 0.05 , comparing vehicle to EX4 using a Tukey test after detecting a significant main effect by one-way ANOVA; $n=16$. C, The number of sucrose pellets earned in the PR operant test was significantly decreased by EX4 VTA microinjection. ${ }^{*} p<$ $0.05,{ }^{* *} p<0.005,{ }^{* * *} p<0.0005$ comparing vehicle to each EX4 dose using the Tukey test after detecting a significant main effect of one-way ANOVA for each time point; $n=15$. Data represent mean \pm SEM. $\boldsymbol{D}, \boldsymbol{E}$, The number of changes in horizontal and vertical (rearing) spontaneous motor activity was measured after VTA directed EX4 injection; $n=11$.
Figure 4. VTA GLP-1R stimulation is sufficient to suppress food intake and reward behavior. The effects of microinjection of EX4 into the VTA on chow intake and PR operant conditioning for sucrose were tested. $A$, Graphical representation (left) and representative tissue section (right) showing VTA microinjection site determined with India ink, used in the study. Aq, aquaduct; $c p$ 
A
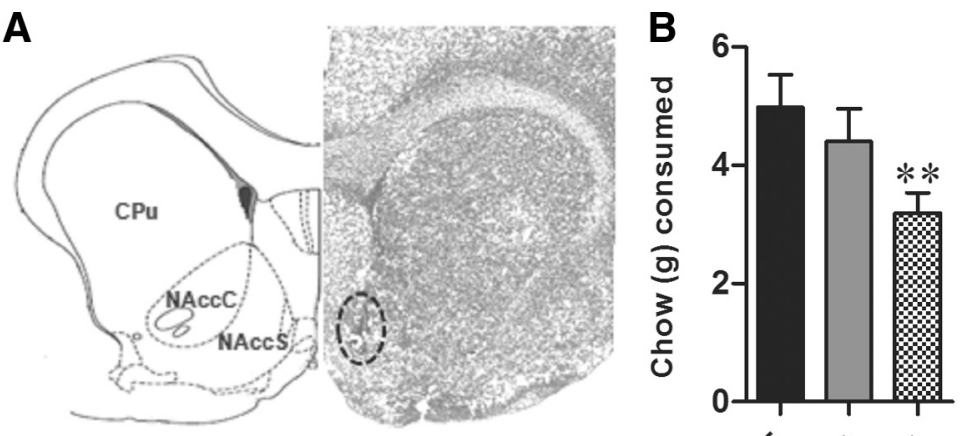

C

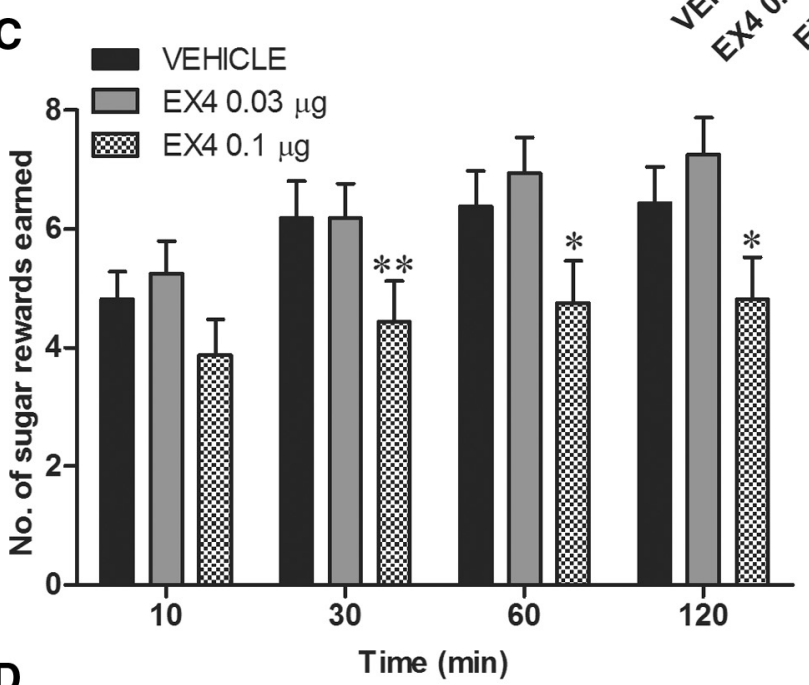

D
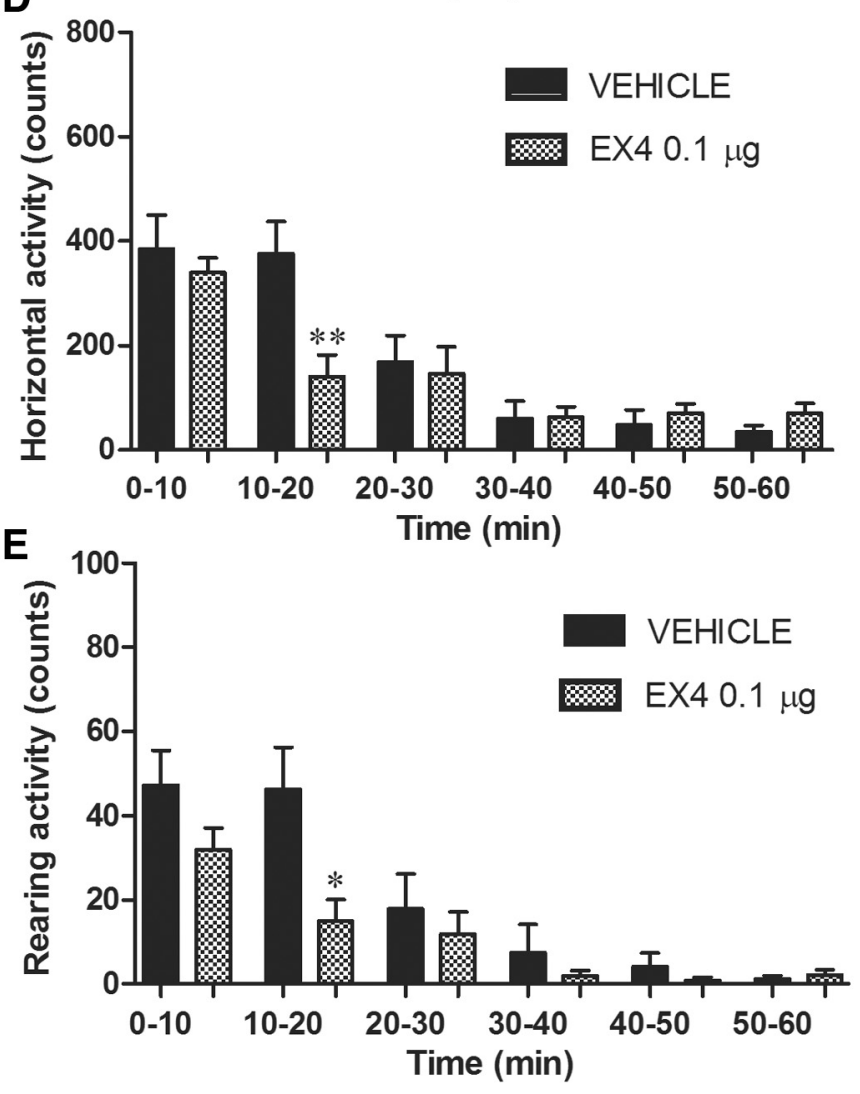

Figure 5. NAc GLP-1Rs contribute to food intake and reward behavior regulation. The effects of microinjection of EX4 into the NAc on chow intake and PR operant conditioning for sucrose were tested. $\boldsymbol{A}$, Graphical representation (left) and representative

\section{Pica response}

VTA and NAc-directed EX4 $0.1 \mu \mathrm{g}$ dose microinjections produced a significant reduction in $24 \mathrm{~h}$ chow intake $\left(F_{(2,19)}=\right.$ $14.4, p<0.0005 ; F_{(2,9)}=5.7, p<0.05$ for VTA and NAc respectively; Figure 8) without producing any significant increase in kaolin consumption. These results indicate that mesolimbic GLP-1R stimulation with EX4, at a dose effective in reducing chow intake, is not accompanied by a malaise response.

\section{Discussion}

In the present study, we provide several lines of evidence for a previously unexplored role of the central GLP-1R in food reward and food motivation. Whereas previous studies implicated homeostatic hypothalamic and brainstem circuits for the anorexigenic effects of GLP-1R stimulation, we now demonstrate a pivotal role for pathways involved in food-reward behavior, especially mesolimbic reward pathways, in this response. We demonstrate that peripheral administration of a long-acting GLP-1 analog, EX4, suppresses food reward and motivation in rats. This is reflected by our observations that EX4 suppresses the ability of chocolate pellets to condition a place preference and also has a suppressive effect on how hard a rat is willing to work for a sweet reward. These effects are exerted centrally as direct injection of EX4 into the brain ventricles induces a dose-dependent suppression of motivated behavior for a sucrose reward in the operant conditioning paradigm. Specifically, we identify key reward nodes, the VTA and NAc, as direct parenchymal targets of GLP-1R stimulation, as microinjection of EX4 into these areas is sufficient to reduce food-motivated behavior. We confirm mediation via the GLP-1R in these effects as a selective

\section{$\leftarrow$}

tissue section (right) showing the NAc microinjection site determined with India ink, used in the study. $\mathrm{CPu}$, caudate and putamen; NAccC, nucleus accumbens core, NAccS, nucleus accumbens shell. $\boldsymbol{B}, \mathrm{NAc}$ microinjection of EX4 produced chow anorexia. ${ }^{* *} p<0.005$, comparing vehicle to each EX4 dose using the Tukey test after detecting a significant main effect by one-way ANOVA; $n=15$. C, The number of sucrose pellets earned in the PR operant test was significantly decreased by EX4 NAc microinjection. ${ }^{*} p<0.05,{ }^{* *} p<0.005$ comparing vehicle to each EX4 dose using the Tukey test after detecting a significant main effect of one-way ANOVA for each time point; $n=16$. Data represent mean \pm SEM. $\boldsymbol{D}, \boldsymbol{E}$, The number of changes in horizontal and vertical (rearing) spontaneous motor activity was decreased only 10-20 min after NAc EX4 injection and unaltered for the remaining $50 \mathrm{~min}$ of measurement. ${ }^{*} p<0.05,{ }^{* *} p<0.005$ comparing vehicle to EX4 using Student's $t$ test; $n=16$. 


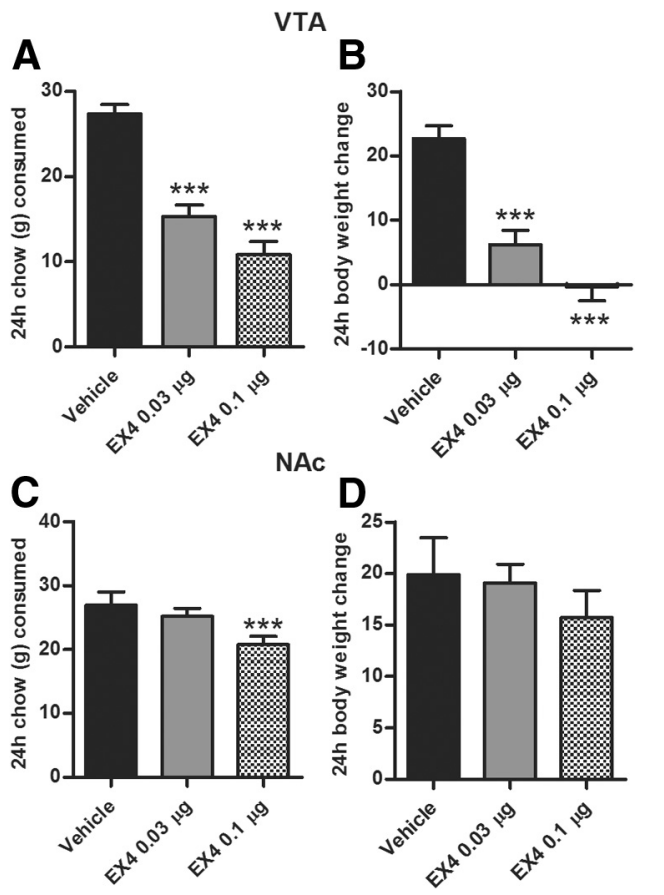

Figure 6. VTA and NAc GLP-1R activation effects on $24 \mathrm{~h}$ chow intake and body weight. VTA GLP-1R activation potently reduced chow intake $(\boldsymbol{A})$ and body weight gain $(\boldsymbol{B})$. In contrast, NAC shell GLP-1 activation produced a small reduction in $24 \mathrm{~h}$ chow intake $(\boldsymbol{C})$ and did note alter body weight gain $(\boldsymbol{D}) .{ }^{* * *} p<0.0005$ comparing vehicle to each EX4 dose using the Tukey test after detecting a significant main effect of one-way ANOVA for each time point; $n=15$. Data represent mean \pm SEM.

GLP-1R antagonist abolished the effects of EX4 on food-motivated behavior. These data demonstrate that central GLP-1R signaling is necessary for EX4 to exert its effects on food-reward behavior. Collectively, the data presented here identify EX4, the GLP-1R agonist, as a new member of the growing list of homeostatic targets, such as leptin and ghrelin, that have been recently indicated to impact on reward (Fulton, 2010).

IP and ICV administration of EX4 potently reduced foodreward behavior. However, such a broad route of drug application that allows for diffusion of drugs to many relevant CNS nuclei leaves the central site of action undetermined. Several brain areas provide likely targets for the effects of EX4 and include brainstem and hypothalamic GLP-1R-expressing nuclei previously shown to play a role in GLP-1 anorexia (Schick et al., 2003; Hayes et al., 2009) that project to the mesolimbic system (e.g., VTA). In fact, for another peripheral hormone, insulin, despite the presence of insulin receptors in the VTA, only the hypothalamic stimulation was effective in reducing food reward (Figlewicz et al., 2008). However, our data showing a suppression of operant responding with a direct VTA or NAc EX4 microinjection demonstrate a direct impact of EX4 on these mesolimbic areas. These data are strengthened by our observation that a dose of EX4 that was ineffective at reducing operant responding when applied ICV was effective when applied to the VTA. Although we cannot entirely eliminate the possibility of some injection liquid diffusing outside of the VTA and NAc borders, several attempts have been made to limit this possibility and address this concern. Small injection volume of $0.5 \mu$ l, previously shown to stimulate mesolimbic NAc shell and separately core selectively (Dossat et al., 2011), was used. The injection sites were confirmed postmortem using India ink, diffusion of which was carefully analyzed. Last, a control experiment was performed in which EX4 at the
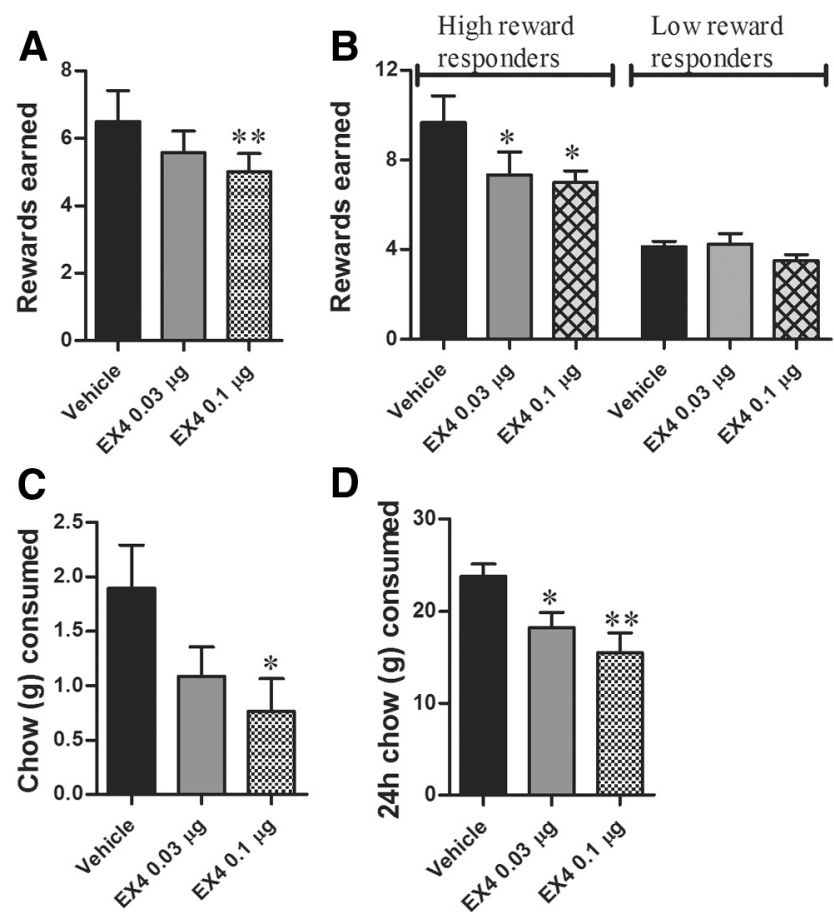

Figure 7. VTA GLP-1R stimulation in overnight ad libitum-fed rats is sufficient to suppress food intake and reward behavior. The effects of microinjection of EX4 into the VTA on chow intake and PR operant conditioning for sucrose in satiated rats were tested. $\boldsymbol{A}$, The number of sucrose pellets earned in the PR operant test was significantly decreased by EX4 VTA microinjection. $\boldsymbol{B}$, Number of rewards earned by the satiated animals analyzed by dividing them into those with a high motivation to work for sucrose at their satiated baseline (on the vehicle condition earned 6 or more rewards; $n=6$ ) and those displaying a low motivation for sucrose (on vehicle earned 5 or less rewards; $n=8$ ). These data might indicate an enhanced sensitivity to the reward reducing effects of EX4 in high reward responders, i.e., those rats are highly motivated to earn sucrose despite their satiated state. $\boldsymbol{C}, \boldsymbol{D}$, VTA microinjection of EX4 reduced free-feeding on chow at both the $1 \mathrm{~h}$ and the $24 \mathrm{~h}$ time points. ${ }^{*} p<0.05,{ }^{* *} p<0.005$ comparing vehicle to each EX4 dose using the Tukey test after detecting a significant main effect of one-way ANOVA for each time point; $n=14$. Data represent mean $\pm \mathrm{SEM}$.

highest dose applied to the parenchyma was injected $2.0 \mathrm{~mm}$ dorsal from the target site (VTA); no effects of the offsite injections were observed, providing further support for very limited diffusion of the drug in the tissue.

Cells in both the VTA and the NAc clearly express the GLP-1R and receive GLP-1-positive fibers likely coming from the NTS (Merchenthaler et al., 1999; Rinaman, 2010; Alhadeff et al., 2012). The VTA contains dopaminergic neurons which innervate the NAc, a pathway suggested to play an integral role in reward from food and addictive drugs. Interestingly, recent data suggest that nearly $50 \%$ of the VTA dopamine neurons express GLP-1Rs (Toth et al., 2011), providing an anatomical foundation for the direct action of EX4 on the VTA dopaminergic neurons. The VTA dopaminergic cells project widely throughout the mesocorticolimbic system. Although some metabolic signals that impact on food reward target the VTA-NAc projecting neurons [for example, ghrelin (Abizaid et al., 2006; Jerlhag et al., 2007)], recent evidence suggests that some metabolic signals target nonaccumbal projecting dopamine neurons. The fat-derived hormone leptin binds to amygdala-projecting VTA dopamine neurons (Leshan et al., 2010), whereas the hypothalamic anorectic peptide, melanocortin, primarily targets the dopaminergic cells projecting to cortical regions (Davis et al., 2011). Given this variety of possible projection sites, the dopamine targets of VTA GLP-1R should be explored in future studies. 

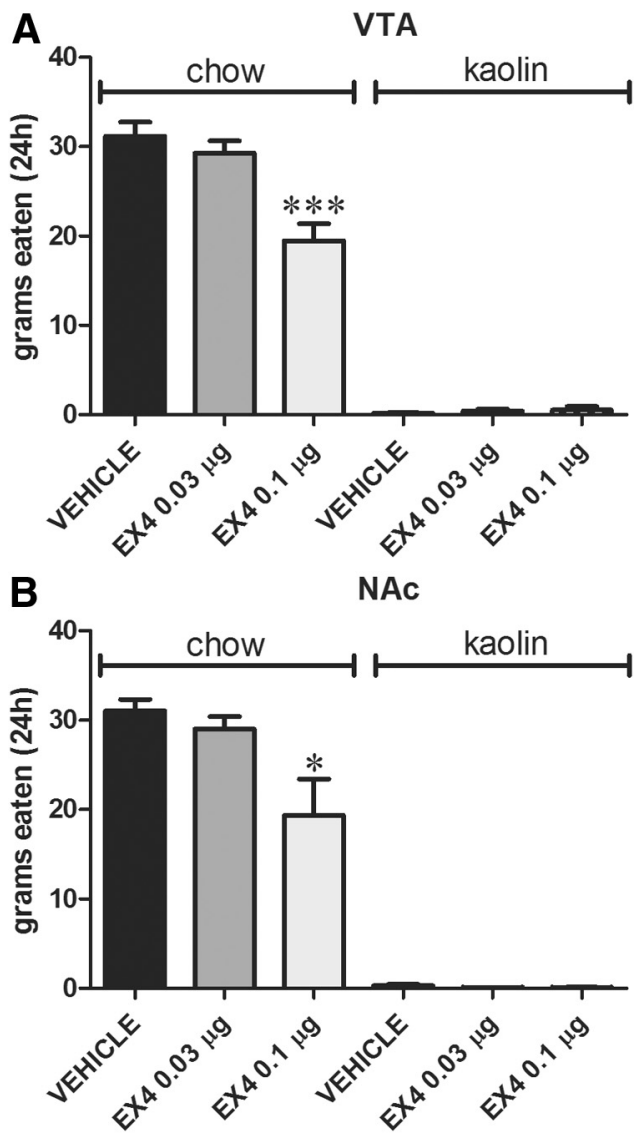

Figure 8. EX4 VTA $(\boldsymbol{A})$ and NAc $(\boldsymbol{B})$-directed microinjection-induced anorexia is not accompanied by a malaise response, as $E X 4$ dose effective in producing a reduction in chow intake did not simultaneously increase kaolin consumption. ${ }^{*} p<0.05,{ }^{* * *} p<0.0005$ comparing vehicle to each EX4 dose using the Tukey test after detecting a significant main effect of one-way ANOVA for each time point; $n=4-8$. Data represent mean \pm SEM.

In addition to food reward, selective GLP-1R stimulation in the VTA and the NAc reduced free-feeding on chow at $1 \mathrm{~h}$ and at $24 \mathrm{~h}$. At $1 \mathrm{~h}$, EX4 reduced chow intake to nearly $50 \%$ of control intake, indicating an important contribution of GLP-1Rs in VTA and NAc to free-feeding behavior. At $24 \mathrm{~h}$ after injection, the VTA GLP-1R activation still potently $(>50 \%)$ reduced chow intake and body weight gain at both doses tested, whereas NAc shell injection produced a small effect only on chow intake at the highest dose tested, potentially indicating differential sensitivity of those two sites to anorexic effects of GLP-1 stimulation. These results contrast with those obtained for insulin, in which a reduction in free-feeding and food-motivated behavior was modulated by a disparate receptor population (Figlewicz et al., 2008). Thus, the mesolimbic GLP-1Rs can contribute to both free-feeding and motivated behavior for palatable food. Interestingly, both our data and those of Alhadeff et al. (2012) suggest that VTA activation of GLP-1Rs is more effective at reducing feeding behavior compared with NAc shell. Also, the NAc core seems to be more sensitive to the anorexic effects of GLP-1, because small doses of GLP-1 that reduced chow intake when injected into NAc core were not effective when applied to the NAc shell (Dossat et al., 2011). Physiological relevance of the GLP-1R activation with the endogenous ligand in the VTA (Alhadeff et al., 2012) and the NAc core (Dossat et al., 2011) is suggested by blockade of GLP-1Rs in those sites leading to elevated free-feeding behavior. In contrast, the GLP-1Rs in the shell do not seem to be required for free- feeding behavior. Considering that it is the shell rather than the core region that may be crucial for the control of motivated behavior, the possibility still remains that NAc shell GLP-1Rs contribute to the motivational aspect of feeding rather than the free-feeding.

In contrast to the effects of EX4 microinjection into the VTA, NAc EX4 reduced spontaneous locomotor activity, which could indicate a reduction of dopamine release or alternatively a reduction of activity of neurons targeted by dopamine in the NAc, e.g., the GABAergic medium spiny neurons. Thus, GLP-1R could be located presynaptically on dopaminergic inputs from the VTA or postsynaptically on dopamine projections.

When a substance emerges as an anorectic agent, it is important to establish whether the reduction in food intake is related to activation of satiety mechanisms or mechanisms involving illness/nausea. A considerable body of literature exploring the potential of GLP-1R stimulation to induce illness exists and indicates that the actions on GLP-1R to alter food intake and produce malaise are dissociable. The potential aversion effects of GLP-1 stimulation have been primarily ascribed to GLP-1Rexpressing neurons in the central nucleus of the amygdala, as GLP-1R blockade in this area attenuates lithium chlorideinduced illness (Kinzig et al., 2002). GLP-1 stimulation in other CNS sites [for example, the hypothalamus (McMahon and Wellman, 1998)], does not induce taste aversion responses. Furthermore, current data indicate that the anorexic effect of GLP-1 stimulation in VTA and NAc is not accompanied by a malaise response. The lack of malaise response from mesolimbic EX4 application shown here is consistent with recent findings by $\mathrm{Al}$ hadeff et al. (2012).

The brainstem GLP-1-producing neurons send projections to both VTA and NAc (Rinaman, 2010; Alhadeff et al., 2012) and potentially contribute to the regulation of reward behavior. Nearly a third of NTS preproglucagon neurons project to the VTA and $46 \%$ to the NAc shell, providing a source of the endogenous agonist for the mesolimbic targets tested here. Importantly, in addition to endogenous GLP-1, the GLP-1 agonist used here, EX4, is now in widespread use as a therapy for diabetic patients. This drug is applied peripherally and can cross the blood-brain barrier (Kastin and Akerstrom, 2003). Thus, the impact of peripherally applied EX4 on reward might be of clinical interest considering this large patient population. It is also striking that a major impact of gastric surgery is to elevate GLP-1 levels (Beckman et al., 2010). This effect, together with our current data, opens up the possibility for future studies evaluating whether GLP-1 provides a link between reduced food cravings and bariatric surgery.

The data presented here provide clear evidence for an impact of GLP-1R on the mesolimbic reward system, focusing especially on their role in food reward. However, the mesolimbic neurocircuitry also plays a crucial role in reward from alcohol or drugs of addiction. The reduction of food-reward behavior and the direct role of mesolimbic GLP-1Rs in this behavior, shown here, open up a field of future research exploring the potential role for GLP-1 or EX4 in alcohol and drug addiction.

Collectively, the effects of GLP-1R stimulation are consistent with a coordinated action on various aspects of food-oriented behavior from food-motivated behavior, food intake (consummatory responses) to satiation. This multitarget activity likely strengthens the overall impact of GLP-1 on feeding by suppressing several aspects of feeding behavior simultaneously. Our data are consistent with the emerging view that metabolism and the 
reward systems are linked and coregulated, in this case by a GLP1R-dependent mechanism.

\section{References}

Abizaid A, Liu ZW, Andrews ZB, Shanabrough M, Borok E, Elsworth JD, Roth RH, Sleeman MW, Picciotto MR, Tschöp MH, Gao XB, Horvath TL (2006) Ghrelin modulates the activity and synaptic input organization of midbrain dopamine neurons while promoting appetite. J Clin Invest 116:3229-3239.

Alhadeff AL, Rupprecht LE, Hayes MR (2012) GLP-1 neurons in the nucleus of the solitary tract project directly to the ventral tegmental area and nucleus accumbens to control for food intake. Endocrinology 153:647-658.

Beckman LM, Beckman TR, Earthman CP (2010) Changes in gastrointestinal hormones and leptin after Roux-en-Y gastric bypass procedure: a review. J Am Diet Assoc 110:571-584.

Davis JF, Choi DL, Shurdak JD, Krause EG, Fitzgerald MF, Lipton JW, Sakai RR, Benoit SC (2011) Central melanocortins modulate mesocorticolimbic activity and food seeking behavior in the rat. Physiol Behav 102:491-495.

Dossat AM, Lilly N, Kay K, Williams DL (2011) Glucagon-like peptide 1 receptors in nucleus accumbens affect food intake. J Neurosci 31:14453-14457.

Figlewicz DP, Bennett JL, Aliakbari S, Zavosh A, Sipols AJ (2008) Insulin acts at different CNS sites to decrease acute sucrose intake and sucrose self-administration in rats. Am J Physiol Regul Integr Comp Physiol 295:R388-R394.

Fulton S (2010) Appetite and reward. Front Neuroendocrinol 31:85-103.

Göke R, Larsen PJ, Mikkelsen JD, Sheikh SP (1995) Distribution of GLP-1 binding sites in the rat brain: evidence that exendin- 4 is a ligand of brain GLP-1 binding sites. Eur J Neurosci 7:2294-2300.

Hayes MR, Skibicka KP, Grill HJ (2008) Caudal brainstem processing is sufficient for behavioral, sympathetic, and parasympathetic responses driven by peripheral and hindbrain glucagon-like-peptide-1 receptor stimulation. Endocrinology 149:4059-4068.

Hayes MR, Bradley L, Grill HJ (2009) Endogenous hindbrain glucagon-like peptide-1 receptor activation contributes to the control of food intake by mediating gastric satiation signaling. Endocrinology 150:2654-2659.

Hodos W (1961) Progressive ratio as a measure of reward strength. Science 134:943-944.

Holst JJ (2007) The physiology of glucagon-like peptide 1. Physiol Rev 87:1409-1439.

Jerlhag E, Egecioglu E, Dickson SL, Douhan A, Svensson L, Engel JA (2007) Ghrelin administration into tegmental areas stimulates locomotor activity and increases extracellular concentration of dopamine in the nucleus accumbens. Addict Biol 12:6-16.

Kastin AJ, Akerstrom V (2003) Entry of exendin-4 into brain is rapid but may be limited at high doses. Int J Obes Relat Metab Disord 27:313-318.

Kinzig KP, D'Alessio DA, Seeley RJ (2002) The diverse roles of specific GLP-1 receptors in the control of food intake and the response to visceral illness. J Neurosci 22:10470-10476.

Laferrère B, Heshka S, Wang K, Khan Y, McGinty J, Teixeira J, Hart AB, Olivan B (2007) Incretin levels and effect are markedly enhanced 1 month after Roux-en-Y gastric bypass surgery in obese patients with type 2 diabetes. Diabetes Care 30:1709-1716.

la Fleur SE, Vanderschuren LJ, Luijendijk MC, Kloeze BM, Tiesjema B, Adan RA (2007) A reciprocal interaction between food-motivated behavior and diet-induced obesity. Int J Obes (Lond) 31:1286-1294.
Leshan RL, Opland DM, Louis GW, Leinninger GM, Patterson CM, Rhodes CJ, Münzberg H, Myers MG Jr (2010) Ventral tegmental area leptin receptor neurons specifically project to and regulate cocaine- and amphetamine-regulated transcript neurons of the extended central amygdala. J Neurosci 30:5713-5723.

McMahon LR, Wellman PJ (1998) PVN infusion of GLP-1-(7-36) amide suppresses feeding but does not induce aversion or alter locomotion in rats. Am J Physiol 274:R23-R29.

Merchenthaler I, Lane M, Shughrue P (1999) Distribution of pre-proglucagon and glucagon-like peptide-1 receptor messenger RNAs in the rat central nervous system. J Comp Neurol 403:261-280.

Mitchell D, Wells C, Hoch N, Lind K, Woods SC, Mitchell LK (1976) Poison induced pica in rats. Physiol Behav 17:691-697.

Pijnenburg AJ, van Rossum JM (1973) Stimulation of locomotor activity following injection of dopamine into the nucleus accumbens [letter]. J Pharm Pharmacol 25:1003-1005.

Rinaman L (2010) Ascending projections from the caudal visceral nucleus of the solitary tract to brain regions involved in food intake and energy expenditure. Brain Res 1350:18-34.

Schick RR, Zimmermann JP, vorm Walde T, Schusdziarra V (2003) Peptides that regulate food intake: glucagon-like peptide 1-(7-36) amide acts at lateral and medial hypothalamic sites to suppress feeding in rats. Am J Physiol Regul Integr Comp Physiol 284:R1427-R1435.

Schirra J, Göke B (2005) The physiological role of GLP-1 in human: incretin, ileal brake or more? Regul Pept 128:109-115.

Skibicka KP, Hansson C, Alvarez-Crespo M, Friberg PA, Dickson SL (2011) Ghrelin directly targets the ventral tegmental area to increase food motivation. Neuroscience 180:129-137.

Skibicka KP, Hansson C, Egecioglu E, Dickson SL (2012) Role of ghrelin in food reward: impact of ghrelin on sucrose self-administration and mesolimbic dopamine and acetylcholine receptor gene expression. Addict Biol 17:95-107.

Takeda N, Hasegawa S, Morita M, Matsunaga T (1993) Pica in rats is analogous to emesis: an animal model in emesis research. Pharmacol Biochem Behav 45:817-821.

Takhar S, Gyomorey S, Su RC, Mathi SK, Li X, Wheeler MB (1996) The third cytoplasmic domain of the GLP-1 [7-36 amide] receptor is required for coupling to the adenylyl cyclase system. Endocrinology 137:2175-2178.

Thorens B, Porret A, Bühler L, Deng SP, Morel P, Widmann C (1993) Cloning and functional expression of the human islet GLP-1 receptor. Demonstration that exendin- 4 is an agonist and exendin-(9-39) an antagonist of the receptor. Diabetes 42:1678-1682.

Toth K, Abraham H, Hajnal A (2011) Glucagon-like peptide-1 (GLP-1) receptors in the ventral tegmental area of the rat: neuronal distribution and in vivo electrophysiological effects. Soc Neurosci Abstr 37:285.02.

Turton MD, O'Shea D, Gunn I, Beak SA, Edwards CM, Meeran K, Choi SJ, Taylor GM, Heath MM, Lambert PD, Wilding JP, Smith DM, Ghatei MA, Herbert J, Bloom SR (1996) A role for glucagon-like peptide-1 in the central regulation of feeding. Nature 379:69-72.

Zheng H, Lenard NR, Shin AC, Berthoud HR (2009a) Appetite control and energy balance regulation in the modern world: reward-driven brain overrides repletion signals. Int J Obes (Lond) 33 [Suppl 2]:S8-S13.

Zheng H, Shin AC, Lenard NR, Townsend RL, Patterson LM, Sigalet DL, Berthoud HR (2009b) Meal patterns, satiety, and food choice in a rat model of Roux-en-Y gastric bypass surgery. Am J Physiol Regul Integr Comp Physiol 297:R1273-R1282. 